\title{
STABILITY PROPERTIES OF STARS WITH CENTRAL He BURNING*
}

\author{
(Abstract)
}

\author{
M. GABRIEL, S. REFSDAL, and H. RITTER \\ Hamburger Sternwarte, University of Hamburg, F.R.G.
}

Stars with central He-burning have recently been studied in great detail by several groups. One of the most interesting results has been that several static $\left(\varepsilon_{g}=0\right)$ solutions with the same chemical profile sometimes exist for such stars when $M \gtrsim 9 M_{\odot}$ (Lauterborn et al., 1971; Kozlowski, 1971). Which of these solutions the corresponding evolutionary models will choose depends on the evolutionary history of the star. Stability properties of such stars are therefore very important, since instabilities (if they exist) may trigger a transition from one branch of solutions to an other branch.

We have therefore carried out a complete secular stability analysis (including a search for complex eigenvalues) for the sequence of models discussed in Lauterborn et al. (1971, § 5). These models have $M=9 M_{\odot}$, homogeneous He-cores of mass $M_{c}$ and the envelopes have step-profiles with $X=0.302$ between $M_{r}=M_{c}$ and $M_{r}=M_{1}$, and $X=0.602$ for $M_{r}>M_{1} . M_{1}$ was chosen constant $\left(M_{1}=0.203 M\right)$ and $M_{c}$-values between $0.1890 M$ and $0.1910 M$ were considered. In agreement with earlier results we found 3 different static solutions for $M_{c}$-values between $0.1897 M$ and $0.1908 M$. The solution with the lowest value of $T(\log T \approx 3.63)$, and the one with the highest value $(3.82>\log T>3.70)$ were always found to be secularly stable (all real eigenvalues are negative, and all complex eigenvalues have negative real parts). The models with an intermediate value of $T_{\text {eff }}$ were, however, always found to be unstable (one real eigenvalue is positive). For $M_{c}$-values smaller than the triple solution region we found only solutions with low effective temperatures $(\log T \lesssim 3.62)$ and for larger $M_{c^{-}}$ values only high effective temperatures $(\log T>3.82)$. These solutions are always found to be stable. The results mentioned above are consistent with results based on general arguments concerning changes of eigenvalues in turning points of a linear series (Gabriel and Ledoux, 1967; Paczyński, 1972). The present investigation gives, however, more information than one can obtain from such general arguments. The information on the complex eigenvalues can for instance be obtained from a complete secular stability analysis only, as we have done here.

The results obtained above indicate that the branch of intermediate solutions is never reached in evolutionary sequences, and that a transition from the branch with low $T_{\text {eff }}$ to the branch with high $T_{\text {eff }}$ (or vice versa) can only be initiated from a turning point $\left(\partial M_{c} / \partial T_{\text {eff }}=0\right)$ of the linear series.

* This paper was presented by $\mathrm{S}$. Refsdal. 


\section{References}

Gabriel, M. and Ledoux, P.: 1967, Ann. Astrophys. 30, 975.

Kozlowski, M.: 1971, Astrophys. Letters 9, 65.

Lauterborn, D., Refsdal, S., and Roth, M. L.: 1971, Astron. Astrophys. 10, 97.

Paczyński, B.: 1972, Acta Astron. 22, 163. 